\title{
ON THE VANISHING OF THE DUAL STIEFEL-WHITNEY CLASSES OF ORIENTABLE MANIFOLDS
}

\author{
STAVROS PAPASTAVRIDIS
}

\begin{abstract}
ABSTRACr. We will determine for which values of $n$ and $k$ the dual $(n-k)$-Stiefel-Whitney class of all orientable $n$-manifolds vanishes.
\end{abstract}

Introduction. Let $w_{i} \in H^{i}\left(B S O ; Z_{2}\right)$ be the mod-2 $i$-dimensional StiefelWhitney class and let $W_{i} \in H^{i}(B S O ; Z)$ be the integral $i$-dimensional Stiefel-Whitney class, which is defined only for $i$ odd and $i>2$. By "manifold" we mean a compact, closed $C^{\infty}$ manifold. If $M$ is an oriented manifold, then $w_{i}(M)$ and $W_{i}(M)$ are the Stiefel-Whitney classes of the normal bundle of the manifold (i.e., the dual Stiefel-Whitney classes of the manifold). In [3], Massey and Peterson found sufficient conditions on $i$, so that $w_{i}(M)=$ $W_{i}(M)=0$ for all $n$-dimensional orientable manifolds, for a fixed $n$. In the same paper they raise the question whether their condition is necessary too. In this note, we prove that this is really the case.

Our main results are the following two theorems.

THEOREM 1. There exists an orientable n-manifold $M$ such that $w_{n-k}(M) \neq$ 0 , if and only if there exist nonnegative integers $a_{0}, a_{1}, \ldots, a_{r}$ which satisfy the following conditions:

(a) $\Sigma_{j} a_{j}=k$,

(b) $\sum_{j} 2^{j} a_{j}=n$,

(c) $a_{1}$ is even,

(d) if $a_{0}=0$, then the first nonzero $a_{j}$ and its immediate successor $a_{j+1}$ must be even,

THEOREM 2. There exists an orientable $n$-manifold $M$ such that $W_{n-k}(M) \neq$ 0 for $n-k$ odd, if and only if there exist nonnegative integers $a_{0}, a_{1}, \ldots, a_{r}$ which satisfy the following conditions:

(a) $\Sigma_{j} a_{j}=k+1$,

(b) $\sum_{j} 2^{j} a_{j}=n$,

(c) $a_{1}$ is even,

(d) if $a_{0}=0$, then the first nonzero $a_{j}$ and its immediate successor $a_{j+1}$ must be even,

(e) $1<a_{r}<k+1$.

Received by the editors October 26, 1978.

AMS (MOS) subject classifications (1970). Primary 57D20, 57D75.

Key words and phrases. Stiefel-Whitney classes, orientable manifolds. 
The "only if" part of the above theorems was proved in [3] by Massey and Peterson, so we will restrict our attention to the "if" part of the theorems.

Theorem 2 follows immediately from Theorem 1 and the following lemma.

LEMMA 3. Let $n, k$ be positive integers such that $k<n$. Let us assume that there exist nonnegative integers $b_{0}, b_{1}, \ldots, b_{s}$ such that

(a) $\Sigma_{j} b_{j}=k+1$,

(b) $\Sigma_{j} 2^{j} b_{j}=n$,

(c) $b_{1}$ is even,

(d) if $b_{0}=0$, then the first nonzero $b_{j}$ and its immediate successor $b_{j+1}$ must be even,

(e) $1<b_{s}<k+1$.

Then there exist nonnegative integers $a_{0}, a_{1}, \ldots, a_{r}$ such that

(a') $\Sigma_{j} a_{j}=k$,

(b') $\Sigma_{j} 2^{j} a_{j}=n$,

(c') $a_{1}$ is even,

(d') if $a_{0}=0$, then the first nonzero $a_{j}$ and its immediate successor $a_{j+1}$ must be even.

Proof. Given $b_{0}, b_{1}, \ldots, b_{s}$ satisfying conditions (a)-(e), then we define $a_{j}=b_{j}$ for $0<j<s-1$ and $a_{s}=b_{s}-2, a_{s+1}=1$.

Proof of Theorem 2. The "only if" part of the theorem was proved in [3]. As for the "if" part of the theorem, let us assume that conditions (a)-(e) are satisfied. Then, by the previous lemma, conditions (a)-(d) of Theorem 1 are satisfied, so there exists an orientable $n$-manifold $M$ such that $w_{n-k}(M) \neq 0$. Since $w_{n-k}$ is the mod-2 reduction of $W_{n-k}$, it follows that $W_{n-k}(M) \neq 0$.

In order to prove the "if" part of Theorem 1, we will use the results of Brown and Peterson [1]. The following proposition follows from Theorem (4.4) of [1].

Proposition 4. The class $w_{n-k}(M)$ will vanish for all orientable n-manifolds $M$, if and only if the following condition is satisfied:

The element $\chi\left(S q^{n-k}\right) i_{k} \in H^{*}\left(K\left(Z_{2}, k\right) ; Z_{2}\right)$, belongs to the image of the map $S q^{1}: H^{*}\left(K\left(Z_{2}, k\right) ; Z_{2}\right) \rightarrow H^{*}\left(K\left(Z_{2}, k\right) ; Z_{2}\right)$. ( $i_{k}$ is the fundamental class of $K\left(Z_{2}, k\right)$.)

The main technical result of this note is the following.

Proposition 5. The element $\chi\left(S q^{n-k}\right) i_{k}$ does not belong to the image of the map $S q^{1}$ in $H^{*}\left(K\left(Z_{2}, k\right) ; Z_{2}\right)$, if and only if, there exist nonnegative integers $a_{0}, a_{1}, \ldots, a_{r}$, which satisfy the following conditions:
(a) $\Sigma_{j} a_{j}=k$,
(b) $\sum_{j} 2^{j} a_{j}=n$,
(c) $a_{1}$ is even,
(d) if $a_{0}=0$, then the first nonzero $a_{j}$ and its immediate successor $a_{j+1}$ must be even. 
Theorem 1 follows immediately from Propositions 4 and 5. The proof of Proposition 5 will occupy the next section.

The cohomology of $K\left(Z_{2}, k\right)$. In order to prove Proposition 5, we will study in some detail the mod-2 cohomology of the space $K\left(Z_{2}, k\right)$ and especially the action of $S q^{1}$. From now on, we assume that $k>0$, the case $k=0$ being trivial.

First of all, we will need some results of Milnor about the mod-2 Steenrod algebra $A$ (see [4]). Let $R=\left(r_{1}, r_{2}, \ldots, r_{i}, \ldots\right)$ be a sequence of nonnegative integers which are all zero except a finite number. Let $S q^{R}$ be the well-known element of the Milnor basis (see [4, p. 162]). Following D. Kraines (see [2]), we define the excess $e(R)=r_{1}+r_{2}+\ldots$ From Milnor's multiplicative tables (see [4, p. 163]), the following two lemmas follow very easily.

LEMMA 6. Let $R=\left(r_{1}, r_{2}, \ldots\right)$. Then

(a) if $r_{1}$ is even, we have $S q^{1} S q^{R}=S q^{r_{1}+1, r_{2}, r_{3}, \cdots}$;

(b) if $r_{1}$ is odd, we have $S q^{1} S q^{R}=0$.

LEMMA 7. Let $m=M-\Sigma_{i} 2^{i} r_{i}$ be a nonnegative integer. Then $S q^{M} S q^{R}=$ $S q^{m R}+\Sigma_{j} S q^{R_{j}}$, where $R=\left(r_{1}, r_{2}, \ldots\right), m R=\left(m, r_{1}, r_{2}, \ldots\right)$ and $e\left(R_{j}\right)>$ $e(m R)$.

Next we quote a result of $D$. Kraines.

LEMMA 8. If $e(R)>k$ then $S q^{R_{i}}=0$.

Proof. See Proposition 3 of [2].

The next statement is an immediate corollary of the two previous lemmas.

COROllary 9. Let $R=\left(r_{1}, r_{2}, \ldots\right), e(R)=k$ and let $r_{t}$ be the first nonzero term of $R$. Then $S q^{R_{i}}=\left(S q{ }_{i_{k}}\right)^{2^{t}}$ where $S=\left(r_{t+1}, r_{t+2}, r_{t+3}, \ldots\right)$.

Proof. See Corollary 4 of [2].

COROllary 10. Let $R=\left(r_{1}, r_{2}, \ldots\right), e(R)=k$ and suppose that the first nonzero term of $R, r_{t}$, is odd. Let $x=S q^{r_{t+1}, r_{t+2}, \cdots}\left(i_{k}\right)$ and $y=S q^{r_{t-1}, r_{t+1}} \cdots\left(i_{k}\right)$. Then

$$
S q^{1}\left(x^{2^{\prime}-2} y\right)=x^{2^{\prime}}=S q^{R_{i}}
$$

Proof. By Lemma 6 and Corollary 9 we have $S q^{1} y=x^{2}$. It follows easily that $S q^{1}\left(x^{2^{\prime}-2} y\right)=x^{2^{t}}$. From Corollary 9 , obviously $x^{2^{\prime}}=S q^{R_{i_{k}}}$.

COROllaRY 11. Let $R=\left(r_{1}, r_{2}, \ldots\right) e(R)=k$ and let us assume that the second nonzero term $r_{t+1}$ of $R$ is odd. Let $x=S q^{r_{t+1}, r_{t+2}} \cdots\left(i_{k}\right)$ and $y=$ $S q^{r_{t+1}-1, r_{t+2}}, \cdots\left(i_{k}\right)$. Then we have $S q^{1}\left(x^{2^{t}-1} y\right)=x^{2^{t}}=S q^{R_{i}}$.

Proof. By Lemma 6 we have $S q^{1} y=x$, so $S q^{1}\left(x^{2^{t}-1} y\right)=x^{2^{t}}$. From Corollary 9 , it follows that $x^{2^{t}}=S q^{R_{i}}$. 
The next result describes the mod-2 cohomology of $K\left(Z_{2}, k\right)$ in terms of the Milnor basis of the Steenrod algebra.

Definition 12. Let $X=\left\{S q^{R_{i}}: e(R)<k\right\}$.

Proposition 13. $H^{*}\left(K\left(Z_{2}, k\right) ; Z_{2}\right)$ is a polynomial algebra with polynomial generators of the elements of $X$.

Proof. In [5] Serre proved that the mod-2 cohomology of $K\left(Z_{2}, k\right)$ is a polynomial algebra, with polynomial generators of all the elements of the form $S q^{j_{1}} S q^{j_{2}} S q^{j_{3}} \ldots S q^{j_{m-1}} S q^{j_{m}}\left(i_{k}\right)$, where $j_{1}>2 j_{2}, j_{2}>2 j_{3}, \ldots, j_{m-1}>$ $2 j_{m}$, and $\left(j_{1}-2 j_{2}\right)+\left(j_{2}-2 j_{3}\right)+\cdots+\left(j_{m-1}-2 j_{m}\right)+j_{m}<k$. From Lemma 8 and Corollary 9 , it is clear that every one of the generators of Serre can be expressed as a polynomial of elements of $X$, so it is enough to prove that the generators of Serre are as many as the elements of $X$. But that is easy to see by corresponding to the generator of Serre $S q^{j_{1}} S q^{j_{2}} \cdots S q^{j_{m}}\left(i_{k}\right)$, the element of $X, S q^{j_{1}-2 j_{2} j_{2}-2 j_{3}}, \ldots, j_{m-1}-2 j_{m} j_{m}\left(i_{k}\right)$, and this correspondence is one-to-one.

Definition 14. Let $M_{1}=\left\{x^{2^{t}}: x \in X\right.$ and $\left.t>0\right\}$, and $M_{2}=$ all the monomials of elements of $X$, which do not belong to $\left.M_{1}\right\}$.

Our last result, before the proof of Proposition 5, is the following: Let $\left[M_{1}\right]$, [ $M_{2}$ ] be the $Z_{2}$-subspaces spanned by $M_{1}, M_{2}$ respectively.

LemMa 15. Let $m$ be a monomial of elements of $X$. Then $S q^{1} m \in\left[M_{2}\right]$, except for the following cases:

(a) $m=S q^{R_{i}}$, with $R=\left(r_{1}, r_{2}, \ldots\right), e(R)<k-1$ and $r_{1}$ even,

(b) $m=x^{2^{t}-2} y$, with $x, y \in X$ and $S q y=x^{2}$,

(c) $m=x^{2}-1$, with $x, y \in X$ and $S q^{1} y=x$.

Proof. This is an easy corollary of Lemma 6 and Corollaries 9-11. We dispose of all the other monomials by examining separately the following cases:

Case 1. $m$ is monomial which contains more than two variables;

Case 2. $m=S q^{R_{i}}$, with $R=\left(r_{1}, r_{2}, \ldots\right), e(R)<k$ and $r_{1}$ odd;

Case 3. $m=x^{a} y^{b}$, with $a, b>1$ and $x \neq y$;

Case 4. $m=x^{a} y$, with $a>1$ and $S q^{1} y$ is not a power of $x$;

Case 5. $m=x^{a} y$, with $a>1, S q^{1} y=x^{j}$ and $a+j \neq$ power of two;

Case 6. $m=x y$, with $S q^{1} x$ is not a power of $y$, and $S q^{1} y$ is not a power of $x$;

Case 7. $m=x y$, where $S q^{1} x=y^{2}$.

It is assumed above, that $x, y \in X$.

And now we come to our final goal.

Proof of Proposition 5. Milnor proved that $\chi\left(S q^{n-k}\right)=\Sigma S q^{S}$, where the summation extends over all sequences $S=\left(s_{1}, s_{2}, \ldots\right)$ such that $\Sigma\left(2^{j}-1\right) s_{j}$ $=n-k$ (see [4, p. 168]). So, by Lemma 8, we have $\chi\left(S q^{n-k}\right) i_{k}=\Sigma S q s_{i_{k}}$ where the summation extends over all sequences $S=\left(s_{1}, s_{2}, \ldots\right)$ such that $\Sigma\left(2^{j}-1\right) s_{j}=n-k$ and $e(S)<k$. Let us assume that there is a sequence of 
nonnegative integers satisfying conditions (a)-(d). Then, we define the sequence $T=\left(a_{1}, a_{2}, \ldots, a_{r}, 0, \ldots\right)$. Clearly $e(T)<k$, and we have $e(T)=$ $k$ if and only if $a_{0}=0$. Since the term $S q^{T_{i}}$ appears in the summation formula for $\chi\left(S q^{n-k}\right) i_{k}$, it follows from the previous lemma and Lemma 6 , Corollaries 10 and 11 , that $\chi\left(S q^{n-k}\right) i_{k}$ does not belong to the image of $S q^{1}$, because it is one of the exceptional cases described in Lemma 15.

\section{REFERENCES}

1. E. Brown and F. Peterson, Relations among characteristic classes. I, Topology 3 (1964), $39-52$.

2. D. Kraines, On the excess in the Milnor basis, Bull. London Math. Soc. 3 (1971), 363-365.

3. W. Massey and F. Peterson, On the dual Stiefel-Whitney classes of a manifold, Bol. Soc. Mat. Mexicana (2) 8 (1963), 1-13.

4. J. Milnor, The Steenrod algebra and its dual, Ann. of Math. (2) 67 (1958), 150-171.

5. J.-P. Serre, Cohomologie modulo 2 des complexes d'Eilenberg-Mac Lane, Comment. Math. Helv. 27 (1953), 198-231.

Department of Mathimatics, University of Patras, Patras, Grebce 